\title{
Mecanismos de ingreso de las universidades públicas estatales en México: un análisis bajo el concepto de equidad
}

José-Alfonso Jiménez-Moreno

\section{RESUMEN}

El artículo aborda el tema de los mecanismos de ingreso a la educación superior; se centra en un análisis de la manera en que las 35 universidades públicas estatales regulan el ingreso de aspirantes. Para ello, se analiza el problema de los mecanismos de ingreso a las universidades bajo el concepto de equidad, retomando los conceptos libertarios de Rawls y el igualitarismo de Dworking. Se concluye que, en lo general, el uso de mecanismos meritocráticos no abona a la equidad, sino que se orienta únicamente hacia el cumplimiento de la justicia formal; además, se resalta la necesidad de que las universidades públicas estatales establezcan mecanismos orientados a reducir la desigualdad.

Palabras clave: educación superior, equidad educativa, pruebas de admisión, universidades públicas, México. 


\section{Mecanismos de admissão das universidades públicas estatais no México: uma análise sob o conceito de equidade}

\section{RESUMO}

O artigo aborda o tema dos mecanismos de admissão ao ensino superior; centra-se em uma análise da maneira em que as 35 universidades públicas estatais regulam o ingresso de aspirantes. Para isso, se analisa o problema dos mecanismos de admissão às universidades sob o conceito de equidade, retomando os conceitos libertários de Rawls e o igualitarismo de Dworking. Se conclui que, em geral, o uso de mecanismos meritocráticos não favorece a equidade, senão que se orienta unicamente no sentido de cumprir com a justiça formal; além do mais, se ressalta a necessidade de que as universidades públicas estatais estabeleçam mecanismos orientados a reduzir a desigualdade.

Palavras chave: ensino superior, equidade educativa, provas de admissão, universidades públicas, México.

\section{Admission mechanisms of public state universities in Mexico: an analysis under the concept of equity}

\section{ABSTRACT}

This article deals with the issue of admission mechanisms to higher education. It focuses on an analysis of the way in which the 35 state public universities regulate the admission of applicants. To do so, the authors first analyze the problem of university admission mechanisms under the concept of equity, taking up the libertarian concepts of Rawls and the egalitarianism of Dworking. They concluded that, in general, the use of meritocratic mechanisms does not contribute to equity, but is oriented only towards the fulfillment of formal justice; in addition, they highlight how necessary it is for state public universities to establish mechanisms aimed at reducing inequality.

Key words: higher education, educational equity, admission tests, public universities, Mexico. 


\section{Introducción}

El ingreso a la educación superior pública en el nivel licenciatura ha sido un tema de gran relevancia en México, al igual que en otros países (Buendía y Rivera, 2010; García, 2016; Lima, Ávila y López, 2015; Mendoza-Rojas, 2015; Segrera, 2015). El proceso de ingreso de los estudiantes a las aulas de la educación superior ha seguido tradicionalmente un modelo basado en la meritocracia (Silva-Laya, 2012), enfocado en la valoración de conocimientos o habilidades de los aspirantes como indicador principal de decisión.

A nivel internacional, la regulación del ingreso de estudiantes a la educación mantiene el uso de la medición de conocimientos o habilidades específicos mediante pruebas a gran escala (Felicetti y Cabrera, 2018; Torrance, 2017); esto sucede en regiones económicamente desarrolladas - como Asia, Europa y casos concretos, como Estados Unidos-, así como en otras zonas - como Latinoamérica o África- (Geiser, 2017; Hlalele y Alexander, 2012; Torrance, 2017; Jia y Ericson, 2016). Si bien en ocasiones se consideran otros aspectos como parte del proceso de ingreso a la educación superior, como el desempeño en niveles educativos previos (Cortés y Palomar, 2008), el ejercicio de la valoración meritocrática mediada por indicadores objetivos enfocados en conocimientos y habilidades se mantiene como el mecanismo hegemónico para regular el ingreso a este nivel educativo.

Este tipo de decisiones suelen centrarse en herramientas orientadas en la valoración de juicios centrados en la objetividad, por ejemplo, se suelen utilizar pruebas tales como el Examen Nacional para el Ingreso a la Educación Superior (EXANI-II) del Centro Nacional para la Evaluación de la Educación Superior (Ceneval, 2019), o el Examen de Habilidades y Conocimientos Básicos (EXHCOBA) (Vera y González-Ledesma, 2018) y en algunos casos, las universidades públicas desarrollan sus propios instrumentos para regular el ingreso, tal es el caso de la Universidad Nacional Autónoma de México (UNAM).
El supuesto en el cual se basa el uso de pruebas objetivas, además de las ventajas técnicas y psicométricas que puede representar - las cuales no son objeto de discusión en la presente propuesta-, se basa en la posibilidad de utilizar métodos justos (Hernández et al., 2018), en los cuales sean las propias habilidades y conocimientos de los sustentantes los indicadores que determinen la posibilidad de ingreso de cualquier aspirante a la educación superior pública.

La premisa de un mecanismo de ingreso justo, basado en la meritocracia, resulta un problema importante poco atendido en el sistema educativo mexicano. La meritocracia implica necesariamente que las condiciones de preparación de niveles educativos previos - los cuales orientan el desarrollo de habilidades y la adquisición de conocimientos que le son evaluados a los sustentantes en este tipo de pruebas - son relativamente homogéneos. El proceso se complejiza cuando las condiciones sociales, de formación y de contexto de los aspirantes son heterogéneas, ya que la universidad se convierte en un "filtro social" (Guzmán y Serrano, 2011: 37), en el que aquellos sustentantes con condiciones contextuales y de formación previos a la licenciatura son más favorables, podrán tener una mayor probabilidad de acceso a estudios superiores.

Este argumento ha sido constatado por diversos estudios en el contexto mexicano (por ejemplo, García, 2016; Guzmán y Serrano, 2011; Piñero, Juárez y Chaín, 2011); sin embargo, es un problema manifiesto en diversas regiones del mundo. Por ejemplo, en Estados Unidos es conocido el uso de la famosa prueba de aptitud académica (SAT), que tiene una historia de intención de medida de gran calado, pero que ha sido objeto de discusiones sobre la manera en que los puntajes parecen beneficiar a poblaciones de raza blanca (Geiser, 2017). Otro ejemplo es China, en el que las pruebas de admisión utilizadas han sido objeto de crítica debido a que los puntajes suelen beneficiar a estudiantes de zonas de alto desarrollo económico, poniendo en desventaja a personas provenientes de provincias o regiones rurales (Jia y Ericson, 2016). 
La preocupación respecto a la manera en que la aplicación de instrumentos o pruebas objetivas basadas en la meritocracia también es manifiesta en otros espacios caracterizados por una mayor inequidad social, como Brasil (Felicetti y Cabrera, 2018); Chile (Donoso y Hawes, 2000); África (Hlalele y Alexander, 2012) y, por supuesto, México (García, 2016; Guzmán y Serrano, 2011; Piñero, Juárez y Chaín, 2011), apuntando incluso que la evaluación del ingreso bajo instrumentos objetivos se consolida como la tecnología de la exclusión, reproduciendo esquemas de distribución inequitativa en términos económicos y sociales (Torrance, 2017).

Este problema no es menor, no sólo por la atención al derecho a la educación superior (UNESCO, 2005), el cual se reconoce en el plano internacional, afirmando que es un modo en que las poblaciones menos favorecidas pueden tener acceso a oportunidades de desarrollo económico y social (Felicetti y Cabrera, 2018); sino también por los cambios en materia de política educativa en México, la cual, a partir de las reformas en 2019, establece la obligatoriedad de la educación superior por parte del Estado mexicano (Diario Oficial de la Federación (DOF), 2019a). $\mathrm{Al}$ respecto, el artículo 48 de la Constitución Política de los Estados Unidos Mexicanos enuncia:

La obligatoriedad de la educación superior corresponde al Estado, el cual la garantizará para todas las personas que cumplan con los requisitos solicitados por las instituciones respectivas. Para tal efecto, las políticas de educación superior estarán basadas en el principio de equidad entre las personas, tendrán como objetivo disminuir las brechas de cobertura educativa entre las regiones, entidades y territorios del país, así como fomentar acciones institucionales de carácter afirmativo para compensar las desigualdades y la inequidad en el acceso y permanencia en los estudios por razones económicas, de género, origen étnico o discapacidad (DOF, 2019b: 14).
El uso de mecanismos meritocráticos basados en la medición de conocimientos y habilidades a través de pruebas a gran escala son altamente aceptados por la sociedad, sin embargo, resulta necesario discutirlos en función de la equidad, dada la relevancia que este concepto tiene en el aseguramiento del derecho a la educación (Alcántara, 2014). La justicia y la equidad, así como el papel que la educación juega en su atención, deben ser tema prioritario en la sociedad actual, particularmente en el sistema educativo. Le corresponde a la escuela la promoción de mejores condiciones sociales y de vida, entre ellas, la reproducción de prácticas de equidad para los sectores de jóvenes mexicanos menos favorecidos (Jiménez, 2017).

Considerando esta problemática y la intención del Estado mexicano de implementar la obligatoriedad de la educación superior, el presente artículo tiene por objetivo analizar los mecanismos de ingreso en las universidades públicas estatales (UPE) en México bajo la óptica conceptual de la equidad, con el fin de aportar al debate sobre la manera en que la regulación del ingreso a la formación profesional pública en el país abona o no al fomento de procesos equitativos de acceso a la educación. Para lograr este objetivo, inicialmente se describe de forma general la manera en que la literatura especializada argumenta el uso de pruebas como mecanismo regulatorio para el ingreso a la educación superior. Posteriormente, se discute la perspectiva de equidad en educación, haciendo uso de la perspectiva liberalista de Rawls y el igualitarismo de Dworking como marco interpretativo de dicho problema. Una vez hecho eso, se describen de manera general los mecanismos de ingreso de las UPE y se analizan a partir de la perspectiva conceptual mencionada, finalizando con algunas reflexiones finales para abonar a la discusión de este tipo de mecanismos y posibilidades de acercamiento a la perspectiva liberalista e igualitaria. 


\section{El problema de los mecanismos regulatorios para el ingreso a la educación superior}

La implementación de mecanismos para regular el ingreso a estudios superiores suele argumentarse en función del incremento en la demanda de este nivel de estudios, derivado de las políticas de masificación de la educación superior (Brunner, 2012; Chaín, Cruz, Martínez y Jácome, 2003; Didou, 2011; Enciso-Ávila y Planas-Coll, 2018; Ordorika, 2014; Silva-Laya, 2012; Villalobos et al., 2017). Como una forma de enfrentar los procesos de incremento de demanda de los estudios superiores que se da desde los años ochenta (Cendrós et al., 2008; Guzmán y Serrano, 2011), las instituciones de educación superior (IES) adoptaron procesos de selección basados en la transparencia (Michel y Pollard, 2020), resaltando como criterio primordial el uso de algún instrumento que permitiera estimar que quienes tuvieran resultados satisfactorios tendrían una mayor probabilidad de éxito en sus estudios universitarios (Chaín et al., 2003; Montero, 2010).

Cabe mencionar que este medio de regulación de ingreso a la educación superior derivado del incremento de la demanda no es exclusivo de México; es un fenómeno que se manifiesta a nivel mundial. Ello se debe, entre otras razones, a la globalización e intercambio tecnológico, que funcionan como medida de presión en términos de competitividad internacional para los sistemas educativos (Jia y Ericson, 2016).

En el contexto de la educación superior a nivel internacional en las últimas décadas, existe una estrecha relación de los estudios superiores con las demandas mercantiles, las capacidades de los jóvenes se valoran como una herramienta de desarrollo y progreso (Villaroel, 2014). De esta manera, los mecanismos de regulación del ingreso permiten no sólo hacer frente al problema de la demanda, también se relacionan con la idea de eficiencia de la educación superior, en la cual quienes ingresan deben representar una potencial de egreso (López et al., 2010).
Esto se torna de particular importancia en las UPE, ya que son las que soportan el mayor peso de formación profesional de la sociedad y son la mayor opción de formación profesional para jóvenes mexicanos (Rodríguez-Gómez, 2015).

En esta intención de regulación de la oferta y con el objetivo de asegurar la eficiencia del proceso formativo, centrarse en la meritocracia pareciera la opción más transparente y justa (Michel y Pollard, 2020; Villaroel, 2014), ya que permiten a las universidades, entre ellas a las UPE, tomar decisiones transparentes sobre el ingreso a sus aulas. De esta manera, se ha entendido que las políticas para el ingreso a la educación superior se reducen a problemas de competencia entre estudiantes en el proceso de admisión; así, relacionan la justicia social en el ingreso a las IES en la noción de igualdad de oportunidades en las cuales se fundamenta la idea de meritocracia (Villalobos et al., 2017). Por tanto, las instituciones que utilizan este tipo de mecanismos se fundamentan en la igualdad de condiciones de los procesos de evaluación como una manera de asegurar la igualdad de condiciones que orillen a una toma de decisiones adecuada.

De esta manera, las IES públicas depositan en la meritocracia la idea de justicia que les permite transparentar las decisiones de ingreso a sus aulas (Delgado et al., 2018); es decir, se aseguran de que todos los estudiantes demuestren con sus propios méritos, en igualdad de condiciones de evaluación, que pueden ingresar a la educación superior. Bajo esta intención, los instrumentos a gran escala han funcionado como una herramienta justa, en la que se cuida un diseño válido y confiable que favorezca la toma de decisiones (Wikström y Wikström, 2020). Este tipo de pruebas se diseñan, así, con la intención de medir en los aspirantes aquellos conocimientos o habilidades que posibilitan un egreso exitoso; en ese sentido, de acuerdo con Geiser (2017), una de las mayores ventajas de este tipo de pruebas recae en la intención de validez predictiva que ofrecen. 
A nivel internacional este tipo de instrumentos son altamente utilizados, tal es el caso de Estados Unidos, Inglaterra, China e India; en estos países hacen uso de pruebas a gran escala como medio regulatorio del ingreso, basados siempre en la medición de habilidades bajo el supuesto de la meritocracia. Existen ejercicios en otros países, como Luxemburgo, Dinamarca y Holanda, donde, además, pueden considerar otros requisitos, como cursos preparatorios para la formación profesional (Niessen y Meijer, 2020). En Latinoamérica resalta el caso chileno, que cuenta con sistema de selección único, que ordena a los postulantes en función de su desempeño en pruebas de admisión (Rodríguez y Padilla, 2017), mientras que, en México, los instrumentos de selección varían en función de cada IES, como más adelante se describe.

Como se ha argumentado, el uso de pruebas a gran escala como mecanismo de ingreso a la educación superior no es exclusivo de México, y si bien a nivel internacional ha sido una respuesta operativa frente a las políticas de masificación de la educación superior desde los años ochenta (Cendrós et al., 2008), su uso incrementó el problema de desigualdad al ingreso a este nivel de estudios (Guzmán y Serrano, 2011). Bajo la idea de justicia y el uso de mecanismos meritocráticos como forma de su implementación, las IES mexicanas se han convertido en un medio en el cual se reproducen inequidades sociales (Guzmán y Serrano, 2011).

A pesar de que, en el discurso psicométrico, los estándares de desarrollo de pruebas de la American Educational Research Association, American Psychological Association y la National Council on Measurement in Education, establecen que quienes se dedican al desarrollo de estos instrumentos deben vigilar los posibles efectos adversos de los puntajes en determinados grupos sociales minoritarios (Geiser, 2017), esta advertencia no es un elemento suficiente para la reducción del problema. Como lo externan Donoso y Hawes (2000: 17): "La discusión se hace más compleja por cuanto la psicometría otorga un viso de objetividad y certeza a sus afirmaciones, haciéndolas extensibles al resto de la realidad social y cultural. La psicometría, sin embargo, no está libre de su condicionamiento histórico-cultural, aparte de los propiamente científicos".

La intención del Estado mexicano de incrementar la cobertura en educación superior es algo que data desde hace décadas y, a través de los años, se ha dado un crecimiento en la matrícula de este nivel educativo. Desde los años cincuenta, México empezó a registrar un aumento en matrícula no regulado (Buendía, 2020), pasando su matrícula de 32498 estudiantes a 4561792 en 2017, incluyendo educación a distancia y posgrado (Martínez, 2020). En las últimas cifras reportadas por la Asociación Nacional de Universidades e Institutos de Educación Superior (ANUIES, 2020), la matrícula en el ciclo 2018-2019 alcanza un total de 4931200 estudiantes. Este fenómeno de incremento de cobertura se traduce en un incremento de 140 veces su tamaño entre 1950 y la actualidad (Tuirán, 2019). Sin embargo, esto no necesariamente se traduce en una satisfacción de la demanda (Buendía y Rivera, 2010), o bien, en un acceso equitativo a la educación superior (Acosta, 2014; Enciso-Ávila y Planas-Coll, 2018; Silva, 2019); a pesar de esta expansión, las oportunidades de ingreso a la formación profesional siguen distribuyéndose en función del origen social (Silva, 2020). Incluso, no existen evidencias claras de que las políticas de los últimos 30 años hayan tenido un impacto directo en la cobertura y aprendizaje de los estudiantes (Buendía, 2020), de tal suerte que el incremento de la matrícula - aparentemente inercial - promueva que en el año 2030 solo $30 \%$ de la matrícula de nivel licenciatura corresponda a personas con alguna desventaja económica (Tuirán, 2019).

Frente a esta situación, "el mejor modo de contribuir a la equidad es dar acceso a los estudiantes a programas pertinentes y de calidad en los que puedan tener posibilidades de éxito" (Tuirán, 2019: 173); sin embargo, la regulación del ingreso a las 
universidades públicas a través de mecanismos de evaluación que aparentemente se fundamentan en la justicia ha traído como consecuencia la exclusión de determinados aspirantes provenientes de grupos sociales, como personas con condición de migrante e indígena en zonas rurales sin oferta pública (EncisoÁvila y Planas-Coll, 2018). En este sentido, a partir de los años noventa comenzó el llamado acceso a las minorías en educación superior y, en consecuencia, se ha fomentado un interés porque las personas de grupos tradicionalmente excluidos participen en la educación superior. Como referente de ello, en 1992, $10 \%$ de la matrícula se conformaba de estudiantes con situación de desventaja económica, mientras que en 2016 ese número se había incrementado a 23\% (Tuirán, 2019). Así, el problema de acceso a la educación por parte de grupos con desventajas se relaciona, en parte, con políticas orientadas al incremento de eficiencia y eficacia a través de mecanismos de evaluación (Salas, Buendía y Pérez, 2019), cuestiones de infraestructura; pero además, se debe apuntar el uso de la meritocracia como indicador de decisión para el ingreso a la educación superior: "Tras ese aparente afán democrático, los ideólogos de la meritocracia olvidan o desconocen que las fuerzas sociales del modelo económico dominante se guían por el poder oligárquico que siempre ha buscado la reproducción de los estratos sociales" (Villaroel, 2014: 256).

Es decir, al hacer uso de la meritocracia en el ingreso a la educación profesional, particularmente los conocimientos y habilidades de los aspirantes a través de exámenes o pruebas a gran escala, puede correrse el riesgo de fomentar la reproducción de diferencias sociales. Así, la resolución de pruebas de admisión no sólo se corresponde con las capacidades cognitivas de los estudiantes, sino con las circunstancias socioeconómicas en las cuales las personas se desarrollaron (Geiser, 2017; Villaroel, 2014). De esta manera, los jóvenes de estratos menos favorecidos tienen menos posibilidad de ingresar a la educación superior (Silva, 2019).
Históricamente, la intención del Estado mexicano respecto a la inclusión de individuos pertenecientes a estratos menos favorecidos en el sistema educativo tiene antecedentes en los años treinta, con la fundación del Departamento de Educación Indígena, así como con la creación de la Comisión Nacional para el Desarrollo de los Pueblos Indígenas en los años cuarenta; pero, sobre todo, por la denominación constitucional del país como diverso cultural y lingüísticamente en las reformas de 1992 (Tapia, 2016). Como consecuencia de estas intenciones, se han generado universidades y espacios de estudio orientados a atender a los grupos tradicionalmente excluidos, como las universidades interculturales (Didou, 2011; Silva, 2019), dedicadas a atender a los pueblos originarios y a que éstos puedan acceder a una educación superior pertinente en términos culturales y lingüísticos (Tapia, 2016). Por otra parte, cabe resaltar la implementación en 2014 del Programa de Inclusión y Equidad Educativa de la Secretaría de Educación Pública (SEP) (Mendoza, 2018; SEP, 2014), orientado hacia el fortalecimiento de la educación especial, educación para infantes provenientes de familias jornaleras agrícolas migrantes, atención a la diversidad lingüística, telesecundaria, atención a grupos en situación de vulnerabilidad. También vale la pena resaltar las becas del Programa Nacional de Becas para la Educación Superior (PRONABES) en 2001, que se creó con la intención de mejorar el acceso y permanencia de estudiantes de bajos recursos en las universidades (Tapia, 2016).

El Estado mexicano ha implementado algunos esfuerzos para poder asegurar el derecho a la educación superior de su población, evitando que sectores tradicionalmente excluidos no formen parte de ella. Sin embargo, es preciso resaltar lo que el Instituto Nacional para la Evaluación de la Educación (INEE) apuntaba en 2014:

Para garantizar el ejercicio pleno del derecho a la educación en una realidad tan diversa 
como la nuestra, el Estado debe considerar la situación desigual de los niños, sus familias y comunidades, a fin de diseñar modelos educativos incluyentes y pertinentes que hagan posible que los objetivos de aprendizaje sean alcanzados por el mayor número de estudiantes. Pero no se puede ofrecer lo mismo si se quiere lograr resultados para grupos distintos. La equidad significa trascender la igualdad y aplicar criterios que permitan darle más y diferente a quienes más lo necesitan” (INEE, 2014: 16).

En ese mismo sentido, el organismo que sustituyó al INEE derivado de las reformas suscitadas en 2019, la Comisión Nacional para la Mejora Continua de la Educación (MEJOREDU), apuntaba ya un discurso similar, en el sentido de que la garantía del derecho a la educación implica asegurar la disponibilidad y accesibilidad a la escuela en cada nivel y tipo educativo, pero, además, asegurar su trayectoria ininterrumpida (MEJOREDU, 2020).

La obligatoriedad de la educación superior es un asunto de Estado, desde la manifestación formal de su obligatoriedad en las reformas constitucionales. Mientras que el Estado mexicano logre trabajar bajo el principio de equidad, ello permitirá alcanzar otros derechos (SEP, 2019) y, como tal, representa un reto mayúsculo. Sin embargo, es un tema en el que también debe considerarse una reflexión sobre los mecanismos que utilizan las IES públicas para regular el ingreso a sus aulas; en ese sentido, las universidades deben asumir su papel en temas de combate a la desigualdad (Beltrán-Llavador et al., 2014), y deben considerar que el asunto de justicia social también se relaciona con sus procesos internos (Villalobos et al., 2017), incluyendo, por supuesto, los mecanismos de ingreso de aspirantes a sus aulas.

\section{Justicia, equidad e igualdad}

Los mecanismos de regulación del ingreso a la educación superior se basan en la meritocracia que, como se exponía previamente, presenta el problema de reproducir desigualdades del contexto en el cual se desarrollaron los aspirantes (Villaroel, 2014). Si bien, desde el ámbito de la evaluación y la psicometría, el uso de pruebas basadas en la meritocrática está plenamente justificado bajo el argumento de la justicia (Wikström y Wikström, 2020), las universidades no son entes apolíticos o neutrales, por lo que deben asumir una reflexión seria sobre sus mecanismos regulatorios del ingreso bajo la idea de equidad (Beltrán-Llavador, Íñigo-Bajo y Mata-Segreda, 2014).

En el plano internacional, el tema de la equidad en educación superior se relaciona fuertemente con lo que desde los años noventa afirmaba la Conferencia Regional de Educación Superior de América Latina y el Caribe (CRES) - convocada por la Organización de las Naciones Unidas para la Educación, la Ciencia y la Cultura (UNESCO) - : la educación superior es un bien público social, un derecho humano y una obligación de los Estados (Maldonado-Maldonado y González, 2017; Suárez y Gairín, 2018); esta perspectiva se mantiene en documentos recientes (CRES, 2018). La consideración a nivel internacional de la educación superior como un derecho y un elemento de promoción de la justicia social implica la búsqueda de mecanismos para mejorar las oportunidades de ingreso a este nivel de estudios, esto con la intención de reducción de brechas sociales (Alcántara y Navarrete, 2014).

La preocupación respecto a este tema es latente a nivel internacional. A partir de que es un derecho humano, se establece la necesidad de eliminar la exclusión social, transformando los sistemas educativos para poder hacer frente a la diversidad estudiantil (Echeita y Ainscow, 2011). Esto implica que todos los agentes educativos busquen esquemas para superar las barreras para el acceso, participación y aprendizaje de la población, y adquiere un matiz singular en sistemas educativos tradicionalmente desiguales, como sucede en Iberoamérica (Echeita y Duk, 2008).

Particularmente en México, el tema de igualdad en educación comenzó a discutirse desde los años 
sesenta con Pablo Latapí (Aguilar-Nery, 2017). Bajo la perspectiva de Latapí, la educación era un órgano de justicia distributiva; es decir, una manera de ayudar a la distribución de los recursos (Villalobos et al., 2017). Según Latapí, para considerar una educación justa debían cubrirse cinco criterios de igualdad: ofrecimiento de oportunidades, de acceso, de perseverancia, de rendimiento y reconocimiento de escolaridad (Aguilar-Nery, 2017); éstos siguen siendo relevantes en las discusiones actuales. Ya en los noventa, comenzó a utilizarse el concepto de equidad, el cual, incluso, se incluyó en la Ley General de Educación de 1993, el cual invita a reflexionar sobre la necesidad de compensación frente a las diferencias y deficiencias de los estudiantes (Silva, 2020).

Otros trabajos, como los de Blanco (2006) y Schmelkes (2009) (ambos citados en Suárez y Gairín, 2018), afirmaban ya la relevancia de apuntar la diferencia dentro de los sistemas educativos, dando tratos diferenciados a quienes mayor necesidad presentan. En este sentido, la discusión sobre equidad no sólo considera a las inequidades socioeconómicas, de género, discapacidad, entre otras variables, sino también aspectos como segregación urbana-rural, situaciones de inmigración u otros problemas más complejos, en coincidencia con Latapí, respecto a la intención distributiva. Frente a esta discusión, vale resaltar algunas de las perspectivas teórico-filosóficas más reconocidas en este tema.

Los trabajos que discuten el tema de la equidad en educación suelen referir mayoritariamente a las ideas del filósofo John Rawls como eje analítico. Para Rawls (2002), la idea más básica de justicia es que la sociedad sea un sistema equitativo de cooperación social; esto asume que los ciudadanos son personas libres e iguales y que, además, la sociedad es ordenada. Este fundamento es de crucial importancia, ya que asume: a) que las personas son libres e iguales, b) una necesaria cooperación social (es decir, posibilidad de acuerdo entre iguales), y c) un orden acordado entre iguales. Estas tres ideas se interrelacionan bajo la idea de libertad. Son las personas libres e iguales entre sí, las que pueden establecer reglas de cooperación y participación social, lo que nos lleva a una participación equitativa.

La idea de equidad deviene de la concepción griega de justicia. De acuerdo con Gómez (2019), la equidad es un tránsito de la isonomia (término que refiere a la igualdad formal) hacia lo que los griegos llamaban la epikeia (igualdad sustancial). Este último es el término más cercano a la equidad, que implica matizar la justicia por medio de principios o valores que permiten ponderar las situaciones. Es decir, para una sociedad equitativa no basta la isonomia, que a su vez nos da un orden, sino que requiere el matiz que abona la epikeia. Volviendo a Rawls, la equidad necesitará del matiz situacional y de la participación social que sólo se da cuando los miembros de una comunidad son iguales entre sí.

Es aquí cuando los mecanismos de regulación para ingreso a la educación superior que se basan en la meritocracia se enfrentan a un problema. Si tomamos la perspectiva igualitaria de Rawls, cualquier mecanismo de ingreso, que asume un acuerdo social - un acuerdo en el que se requieren mecanismos para orientar la decisión de quién debe continuar sus estudios profesionales en una universidad pública-, debería requerir que los individuos sean libres y pares; es decir, que sean iguales en condiciones. Esto no es baladí, ya que el papel de los principios de justicia consiste en especificar los términos equitativos y la cooperación social (Rawls, 2002); por lo que si los mecanismos de ingreso a la educación superior basados en la merictocracia no asumen la fragmentación social y la discriminación de grupos minoritarios (Silva 2020), entonces no abonan a una sociedad equitativa, sino que se mantienen sólo en el plano de la justicia formal.

$\mathrm{Si}$ asumimos la perspectiva de Rawls, se deben buscar mecanismos que permitan ofrecer las mismas oportunidades a grupos menos favorecidos, posibilitando así la igualdad de todos los que participan de la 
vida social - la educación como una parte importante de esa vida social- (Cortés, 2005). El problema se acentúa debido a que, como se comentó, las personas que participan en el proceso de ingreso a la educación superior en UPE no necesariamente se enfrentan a este proceso en igualdad de condiciones de desarrollo.

Adicionalmente se debe enfatizar que la perspectiva de Rawls (2002) parte de la idea de libertad; en este esquema, la libertad es la condición inicial para el ejercicio de la equidad. Sin embargo, vale recordar también la perspectiva igualitaria, la cual puede ofrecer una visión más robusta para el análisis de los mecanismos de ingreso a educación superior en México. El igualitarismo - a diferencia del liberalismo de Rawls - propone a la igualdad como valor precedente sobre la libertad; esto es contrario al esquema liberal, que antepone el principio de libertad sobre la igualdad entre personas (Cortés, 2005; Dworking, en Gallego y Bullemore, 2016). De esta manera, no sólo bastará con la libertad de buscar participar en la educación superior en México, sino buscar la igualdad de quienes participan. En ese sentido, las universidades deben asumir el reto de asegurar el ingreso a este nivel educativo de personas provenientes de diversos sectores de la población (Acosta, 2014), manteniendo así una visión cercana de Rawls y complementándola con la perspectiva igualitaria de Dworking. En la consideración de este reto, la discusión sobre mecanismos de ingreso a la educación superior pública equitativos requieren valorar cumplir con el principio de igualdad previo al de libertad.

La igualdad de Dworking no debe entenderse como la intención de supresión del azar, en la cual las personas nacen y se desarrollan bajo condiciones inequitativas de alimentación y salud, y correspondería al Estado la eliminación de las condiciones azarosas; sino como la relevancia en que el Estado mexicano asegure lo que Rawls llama los bienes primarios (Gallego y Bullemore, 2016). Son este tipo de bienes los que permiten que las personas se desempeñen socialmente (libertad, respeto, riqueza, entre otros). La educación superior como derecho (UNESCO, 2005) - que en México está constitucionalmente establecido (DOF, 2019a) - , se vuelve un bien primario necesario que permitirá a las personas acceder a otros bienes.

Bajo estas ideas, los mecanismos que regulan qué personas pueden acceder a la educación superior se basan únicamente en la meritocracia, dan por hecho la igualdad de condiciones, parten de la idea de que la evaluación trata de manera igualitaria a todos los aspirantes; sin embargo, no permiten el matiz de decisión que caracteriza a la equidad (epikeia que argumenta Gómez, 2019). De esta manera, limitan la participación social de la población desfavorecida, reproduciendo así las diferencias de origen que imposibilitan el acceso a bienes primarios y secundarios. En concreto, como lo afirman Maldonado-Maldonado y González (2017), mantener el enfoque meritocrático en el acceso a la educación superior no ayuda a disminuir desigualdades sociales.

En este sentido, para una población tradicionalmente desigual, no basta con la consideración de la libertad de participar en procesos de ingreso, asumiendo condiciones de evaluación equivalentes o igualitarias entre individuos. También se requiere de la reflexión sobre las condiciones de igualdad que anteceden a las posibilidades de libertad de los individuos (Gallego y Bullemore, 2016).

\section{Mecanismos de ingreso a la educación superior en las UPE mexicanas}

Con el fin de complementar el objetivo de este artículo, es importante hacer una revisión de los mecanismos de ingreso de las 35 UPE en México a la luz de los marcos analíticos propuestos. ${ }^{1}$ Para ello, inicialmente se identificaron las convocatorias de ingreso a nivel licenciatura de cada una de estas universidades en cuestión, posteriormente se clasificaron en función

${ }^{1}$ Las 35 instituciones del estudio corresponden a la clasificación establecida por la SEP. El listado oficial de las UPE puede consultarse en la siguiente página web: https://www.gob.mx/sep/acciones-y-programas/universidades-publicas-estatales-educacion-superior. 
de los instrumentos y procesos utilizados. La decisión de realizar el análisis de los mecanismos de ingreso de las UPE mexicanas, se basó en que este tipo de universidades cuenta con financiamiento público federal y estatal (SEP, 2020), con una amplia participación en la matrícula que atiende a nivel nacional (ANUIES, 2020). Existen otro tipo de IES, como las universidades privadas, institutos tecnológicos, escuelas normales y universidades de financiamiento federal (como la UNAM o la Universidad Autónoma Metropolitana (UAM) en la Ciudad de México), las cuales no se consideraron en este estudio; ello debido a que otro tipo de instituciones mantienen factores contextuales y de financiamiento de otra índole, lo cual implica un análisis bajo consideraciones diferentes a las propuestas. Las UPE son una pieza importante en la operación de la educación superior pública en México, sobre todo considerando el nivel constitucional que obliga al Estado mexicano a asegurar dicho derecho en su población. Para poder valorar la relevancia de este tipo de universidades respecto a la matrícula que atiende a nivel nacional, la tabla 1 muestra la matrícula de estas instituciones en el ciclo 2018-2019.

Del total de la matrícula de estudiantes en educación superior en México reportada por ANUIES en el ciclo 2019-2020: 4931200 estudiantes, las UPE representan el 25.64\% del total nacional; además de tener representación en todo el territorio nacional. En ese sentido, las UPE representan un porcentaje considerable de la educación superior mexicana.

Para efectos de este texto, se revisaron las 35 convocatorias de ingreso a estudios de nivel licenciatura de las 35 UPE mexicanas vigentes en 2020. Cabe considerar que sólo se tomaron en cuenta los procedimientos de primer ingreso, no así los trámites para ingresos rezagados $\mathrm{u}$ otras condicionantes, o bien, para programas de otro nivel de estudios, o bien, de modalidad no presencial.

Previo a mostrar las características de los mecanismos de selección, vale describir algunos de los instrumentos mayormente utilizados por las IES en México. El primero de ellos es EXANI-II, esta prueba es desarrollada por el Ceneval y es altamente utilizada, en 2018 alcanzó un total de 772524 sustentantes en todo el país. Si se considera que en 2018 ingresaron un total de 2169817 solicitudes de primer ingreso a nivel licenciatura (ANUIES, 2019), quienes presentaron EXANI-II representan 35\% de dichas solicitudes. El EXANI-II evalúa pensamiento matemático, estructura de la lengua y comprensión lectora, que se consideran las áreas predictivas para valorar el potencial de los aspirantes como estudiantes de nivel profesional (Ceneval, 2019). Otro instrumento es el Examen de Competencias Básicas (EXCOBA) (Métrica educativa, 2020). Asimismo, algunas instituciones - como se verá más adelantehacen uso de la Prueba de Aptitud Académica (PAA) de la empresa College Board, que evalúa lectura, redacción, matemáticas e inglés (College Board, 2020a).

La clasificación de los medios que las UPE mexicanas utilizan para seleccionar a sus aspirantes se realizó en función de la información pública de cada una de estas universidades. Se identificaron diversos mecanismos que, de manera inductiva (Saldaña, 2013), se clasificaron en cinco rubros: a) examen de selección, b) promedio del nivel educativo previo, c) curso propedéutico, d) otro tipo de exámenes y, e) otras condicionantes. La tabla 2 muestra los mecanismos que utilizan cada una de las 35 UPE en México.

En 2020, las 35 UPE mexicanas hicieron uso de un examen de ingreso como herramienta preponderante para la selección de sus estudiantes de nivel licenciatura; $71 \%$ (25) de las universidades hacen uso del EXANI-II del Ceneval, de ese total, 12\% (3) lo utilizan con una ponderación en particular, combinándolo con algún otro examen; el resto lo utiliza como mecanismo preponderante. Por otra parte, 11\% (4) de las UPE hacen uso del EXCOBA; $14 \%$ (5) hacen uso de instrumentos desarrollados en la misma institución, y sólo tres de ellas lo utilizan como mecanismo exclusivo. En menor medida, 5\% (2) de las UPE hacen uso del instrumento PAA ofrecido por la empresa College Board (2020b). 
Tabla 1. Matrícula de educación superior en UPE. Ciclo 2019-2020

\begin{tabular}{|c|c|c|c|c|c|c|}
\hline \multirow{2}{*}{ Universidades públicas estatales } & \multicolumn{5}{|c|}{ Nivel de estudios } & \multirow{2}{*}{ Total general } \\
\hline & $\begin{array}{l}\text { Técnico } \\
\text { superior }\end{array}$ & Licenciatura & Especialidad & Maestría & Doctorado & \\
\hline Benemérita Universidad Autónoma de Puebla & 452 & 81599 & 1278 & 1371 & 621 & 85321 \\
\hline Instituto Tecnológico de Sonora & 75 & 17031 & & 419 & 72 & 17597 \\
\hline $\begin{array}{l}\text { Universidad Autónoma Benito Juárez de } \\
\text { Oaxaca }\end{array}$ & & 19931 & 183 & 542 & 69 & 20725 \\
\hline Universidad Autónoma de Aguascalientes & & 15730 & & 250 & 168 & 16148 \\
\hline Universidad Autónoma de Baja California & & 63728 & 310 & 992 & 498 & 65528 \\
\hline Universidad Autónoma de Baja California Sur & 188 & 7496 & & 137 & 95 & 7916 \\
\hline Universidad Autónoma de Campeche & & 7086 & 153 & 103 & 4 & 7346 \\
\hline Universidad Autónoma de Chiapas & & 22610 & 100 & 471 & 94 & 23275 \\
\hline Universidad Autónoma de Chihuahua & & 27375 & 284 & 1142 & 168 & 28969 \\
\hline Universidad Autónoma de Ciudad Juárez & & 33018 & 300 & 528 & 153 & 33999 \\
\hline Universidad Autónoma de Coahuila & & 25415 & 100 & 1324 & 264 & 27103 \\
\hline Universidad Autónoma de Guerrero & 25 & 34669 & 149 & 648 & 91 & 35582 \\
\hline Universidad Autónoma de Nayarit & 93 & 17841 & 182 & 256 & 65 & 18437 \\
\hline Universidad Autónoma de Nuevo León & 27 & 118193 & 820 & 3056 & 950 & 123046 \\
\hline Universidad Autónoma de Occidente & & 15951 & & 263 & 71 & 16285 \\
\hline Universidad Autónoma de Querétaro & 55 & 21492 & 569 & 1407 & 442 & 23965 \\
\hline Universidad Autónoma de San Luis Potosí & 91 & 29840 & 549 & 1007 & 460 & 31947 \\
\hline Universidad Autónoma de Sinaloa & 394 & 81633 & 1320 & 490 & 311 & 84148 \\
\hline Universidad Autónoma de Tamaulipas & 332 & 36068 & 760 & 591 & 233 & 37984 \\
\hline Universidad Autónoma de Tlaxcala & & 15727 & 17 & 592 & 179 & 16515 \\
\hline Universidad Autónoma de Yucatán & & 15839 & 987 & 506 & 143 & 17475 \\
\hline Universidad Autónoma de Zacatecas & 29 & 25559 & 167 & 1447 & 262 & 27464 \\
\hline Universidad Autónoma del Carmen & & 6294 & & 71 & & 6365 \\
\hline Universidad Autónoma del Estado de Hidalgo & & 31869 & 192 & 517 & 258 & 32836 \\
\hline Universidad Autónoma del Estado de México & 94 & 64114 & 1906 & 790 & 579 & 67483 \\
\hline Universidad Autónoma del Estado de Morelos & & 27292 & 106 & 599 & 454 & 28451 \\
\hline
\end{tabular}




\begin{tabular}{|c|c|c|c|c|c|c|}
\hline Universidad de Colima & & 12848 & 190 & 324 & 142 & 13504 \\
\hline Universidad de Guadalajara & 1763 & 121912 & 2449 & 3033 & 1086 & 130243 \\
\hline Universidad de Guanajuato & 13 & 24982 & 1202 & 1189 & 445 & 27831 \\
\hline Universidad de Quintana Roo & & 5762 & & 94 & 44 & 5900 \\
\hline Universidad de Sonora & & 31727 & 59 & 699 & 305 & 32790 \\
\hline Universidad Juárez Autónoma de Tabasco & 106 & 31669 & 385 & 516 & 189 & 32865 \\
\hline Universidad Juárez del Estado de Durango & & 13864 & 424 & 473 & 105 & 14866 \\
\hline $\begin{array}{l}\text { Universidad Michoacana de San Nicolás de } \\
\text { Hidalgo }\end{array}$ & 2 & 38238 & 103 & 1113 & 556 & 40012 \\
\hline Universidad Veracruzana & 465 & 62115 & 319 & 1166 & 522 & 64587 \\
\hline Total General & 4204 & 1206517 & 15563 & 28126 & 10098 & 1264508 \\
\hline
\end{tabular}

Fuente: elaboración propia con información de ANUIES (2020).

Resaltan algunos casos en los cuales los exámenes se utilizan de manera combinada. Por ejemplo, aquellas UPE que establecen otros exámenes como complementarios a formaciones muy específicas (como letras, artes o un segundo idioma), tal es el caso de la UAA, UABCS, UNACAR, UAEMEX, UMICH, UASLP, UNISON y UV. Incluso en estos casos, resalta que el mecanismo preponderante es el uso de examen de selección estandarizado.

Los exámenes como EXANI-II y EXCOBA, además de los ejercicios realizados por las propias IES (como en el caso de UABC, UMICH y UABJO), parten de la premisa de la meritocracia, en la cual se hace uso de un proceso claro, justo y libre de sesgos para este tipo de decisiones (López et al., 2010; Michel y Pollard, 2020). Prácticamente el total de las UPE hacen uso de algún tipo de instrumento a gran escala, los cuales parten del supuesto de la justicia (Wikström y Wikström, 2020) - en términos formales - para evaluar bajo las mismas condiciones a todos los aspirantes que ejercen su libertad de participación en este tipo de procesos de selección. En lo particular, resalta que más de 90\% de las UPE utilizan instrumentos desarrollados por empresas particulares, lo cual, como se comentó, se presenta como la opción más transparente y justa (Michel y Pollard, 2020); sin embargo, ello también es un tema de búsqueda de legitimidad externa y social, que permite que las universidades se presenten como buenos gestores frente a la sociedad, a las autoridades estatales y federales (Acosta, 2020), más que abonar al deber universitario de reducción de brechas sociales (Guzmán y Serrano, 2011). 
Tabla 2. Resumen de mecanismos de ingreso de las 35 UPE mexicanas

\begin{tabular}{|c|c|c|c|c|c|}
\hline & Institución & $\begin{array}{c}\text { Examen } \\
\text { de selección }\end{array}$ & $\begin{array}{l}\text { Curso } \\
\text { propedéutico }\end{array}$ & $\begin{array}{l}\text { Otro tipo de } \\
\text { exámenes }\end{array}$ & $\begin{array}{l}\text { Otras } \\
\text { condicionantes }\end{array}$ \\
\hline 1 & Universidad Autónoma de Aguascalientes (UAA) & EXANI-II & $\begin{array}{l}\text { Para alumnos } \\
\text { condicionados } \\
\text { en función de sus } \\
\text { resultados }\end{array}$ & $\begin{array}{l}\text { Pruebas de ejecución } \\
\text { para carreras como } \\
\text { música, actuación y } \\
\text { cinematografía }\end{array}$ & \\
\hline 2 & $\begin{array}{l}\text { Universidad Autónoma de Baja California } \\
\qquad(U A B C)\end{array}$ & $\begin{array}{l}\text { Examen desarrollado } \\
\text { en la institución }\end{array}$ & & $\begin{array}{l}\text { Aplicación de } \\
\text { examen psicométrico } \\
\text { (sin fines de } \\
\text { selección) }\end{array}$ & \\
\hline 3 & $\begin{array}{l}\text { Universidad Autónoma de Baja California Sur } \\
\qquad \text { (UABCS) }\end{array}$ & EXANI-II & $\begin{array}{l}\text { Para algunas } \\
\text { ingenierías }\end{array}$ & $\begin{array}{l}\text { Aplicación de } \\
\text { examen psicométrico }\end{array}$ & \\
\hline 4 & Universidad Autónoma de Campeche (UACam) & EXANI-II & & & \\
\hline 5 & Universidad Autónoma del Carmen (UNACAR) & EXANI-II & & Examen de inglés & \\
\hline 6 & Universidad Autónoma de Coahuila (UADEC) & EXANI-II & & & \\
\hline 7 & Universidad de Colima (UCOL) & $\begin{array}{l}\text { EXANI-II (50\% de } \\
\text { ponderación) }\end{array}$ & & & \\
\hline 8 & Universidad Autónoma de Chiapas (UNACH) & EXANI-II & & & \\
\hline 9 & Universidad Autónoma de Chihuahua (UACH) & EXANI-II & & & \\
\hline 10 & Universidad Autónoma de Ciudad Juárez (UACJ) & EXHCOBA & & & \\
\hline 11 & Universidad Juárez del Estado de Durango (UJED) & EXANI-II & & & \\
\hline 12 & Universidad de Guanajuato (UG) & EXCOBA & & & \\
\hline 13 & Universidad Autónoma de Guerrero (UAGro) & EXANI-II & & & $\begin{array}{c}13 \% \text { de los espacios } \\
\text { se destinan para } \\
\text { grupos vulnerables }\end{array}$ \\
\hline 14 & $\begin{array}{l}\text { Universidad Autónoma del Estado de Hidalgo } \\
\qquad(\text { UAEH) }\end{array}$ & EXANI-II & & & \\
\hline 15 & Universidad de Guadalajara (udec) & PAA & & & \\
\hline 16 & $\begin{array}{l}\text { Universidad Autónoma del Estado de México } \\
\text { (UAEMex) }\end{array}$ & EXANI-II & & $\begin{array}{l}\text { Pruebas particulares } \\
\text { en letras } y \text { artes }\end{array}$ & \\
\hline 17 & $\begin{array}{l}\text { Universidad Michoacana de San Nicolás de } \\
\text { Hidalgo }(\mathrm{UMICH})\end{array}$ & $\begin{array}{l}\text { Examen desarrollado } \\
\text { en la institución }\end{array}$ & & $\begin{array}{l}\text { Disciplinas de la salud } \\
\text { aplican el EXANI-II }\end{array}$ & \\
\hline 18 & $\begin{array}{l}\text { Universidad Autónoma del Estado de Morelos } \\
\text { (UAEMOr) }\end{array}$ & EXANI-II & & & \\
\hline 19 & Universidad Autónoma de Nayarit (UAN) & EXANI-II & & & \\
\hline
\end{tabular}




\begin{tabular}{|c|c|c|c|c|c|}
\hline 20 & Universidad Autónoma de Nuevo León (UANL) & EXANI-II & & & \\
\hline 21 & $\begin{array}{l}\text { Universidad Autónoma "Benito Juárez" de } \\
\text { Oaxaca (UABJO) }\end{array}$ & $\begin{array}{l}\text { Examen desarrollado } \\
\text { en la institución }\end{array}$ & & & \\
\hline 22 & $\begin{array}{l}\text { Benemérita Universidad Autónoma de Puebla } \\
\text { (BUAP) }\end{array}$ & PAA & & & \\
\hline 23 & Universidad Autónoma de Querétaro (UAQ) & EXHCOBA & $\begin{array}{l}\text { Para algunos } \\
\text { programas }\end{array}$ & & \\
\hline 24 & Universidad de Quintana Roo (UQROO) & EXANI-II & & & \\
\hline 25 & $\begin{array}{l}\text { Universidad Autónoma de San Luis Potosí } \\
\text { (UASLP) }\end{array}$ & $\begin{array}{l}\text { Examen desarrollado } \\
\text { por la institución } \\
(45 \%) \\
\text { EXANI-II(40\%) }\end{array}$ & & Examen psicométrico & \\
\hline 26 & Universidad Autónoma de Sinaloa (UAS) & EXANI-II & & & \\
\hline 27 & Universidad Autónoma de Occidente (UAdeo) & EXANI-II & & & \\
\hline 28 & Universidad de Sonora (UNISON) & EXCOBA & & $\begin{array}{l}\text { Pruebas particulares } \\
\quad \text { en artes } \\
\text { Examen diagnóstico } \\
\text { de dominio del inglés }\end{array}$ & \\
\hline 29 & Instituto Tecnológico de Sonora (ITSON) & EXANI-II & & & \\
\hline 30 & Universidad Juárez Autónoma de Tabasco (UJAT) & EXANI-II & & & \\
\hline 31 & Universidad Autónoma de Tamaulipas (UAT) & EXANIIII & & & \\
\hline 32 & Universidad Autónoma de Tlaxcala (UATX) & EXANIIII & & & $\begin{array}{l}\text { Se aplica una prueba } \\
\text { de orientación } \\
\text { vocacional }\end{array}$ \\
\hline 33 & Universidad Veracruzana (Uv) & EXANIIII & & $\begin{array}{l}\text { Pruebas particulares } \\
\text { en artes }\end{array}$ & \\
\hline 34 & Universidad Autónoma de Yucatán (UADY) & EXANI-II & & & \\
\hline 35 & Universidad Autónoma de Zacatecas (UAZ) & $\begin{array}{l}\text { Examen desarrollado } \\
\text { por la institución } \\
\text { (ponderación } \\
\text { variada) y EXANI- } \\
\text { "I (ponderación } \\
\text { variada) }\end{array}$ & & & \\
\hline
\end{tabular}

Fuente: elaboración propia con información de UAA (2020), UABC (2020), UABCS (2020), UACam (2020), UNACAR (2020), UADEC (2020), UCOL (2020), UNACH (2020), UACH (2020), UACJ (2020), uJED (2020), UG (2020), UAGro (2020), UAEH (2020), UdeG (2020), UAEMeX (2020), UMICH (2020), UAEMor (2020), UAN (2020), UANL (2020), UABJO (2020), BUAP (2020), UAQ (2020), UQROO (2020), UASLP (2020), UAS (2020), UAdeo (2020), UNISON (2020), ITSON (2020), UJAT (2020), UAT (2020), UATX (2020), UV (2020), UADY (2020) y UAZ (2020). ${ }^{2}$

${ }^{2} \mathrm{Si}$ bien la información consultada durante 2020 mostraba estos datos respecto a los procesos de ingreso, es posible que estos mecanismos hayan tenido algún ajuste posterior a la pandemia derivada del virus SARS-CoV-2. 
De acuerdo con la perspectiva clásica griega (Gómez, 2019), la idea de un instrumento que apela a la justicia formal - que toma a todos por igual - es más acorde con la isonomia y no apela a la igualdad sustancial, es decir, a la epikeia o equidad. Además, como se ha comentado, son medios que reproducen las diferencias sociales; por ende, la decisión de selección de las IES, como una decisión normativa, debe surgir de las circunstancias específicas o condiciones de la población que participa en esta estructura social (Cortés, 2005), lo cual contraviene el amplio uso de exámenes que parten de la perspectiva de la justicia formal, fundamentando su utilización en la sistematización de la evaluación que realizan, como si se tratase de una decisión apolítica.

Algo similar sucede con la consideración del promedio del nivel de estudio previo (educación media superior) como indicador para la decisión de ingreso a las UPE. Todas las IES establecen un promedio mínimo para poder concursar (usualmente mayor a 7, en una escala del 1 al 10); sin embargo, de las 35 UPE, solo la UPCOL considera este indicador con una ponderación de 50\%, además del examen, para decidir sobre el ingreso de los aspirantes. Este indicador es uno de los valores que puede tener mayor peso para ingresar y tener éxito en los estudios profesionales (Guzmán y Serrano, 2011), ya que refleja las condiciones de desarrollo previas al nivel superior. De tal suerte, se corre el riesgo de poner en juego "mecanismos de selección social" (Guzmán y Serrano, 2011: 51), reproduciendo desigualdades (Villaroel, 2014), en las cuales se beneficie a aspirantes con condiciones favorables en niveles previos.

Los instrumentos sistematizados a gran escala utilizados, como el EXANI-II, han sido sujetos a críticas debido a la limitación que tienen al medir conocimientos y habilidades cognitivos fuera del contexto de los aspirantes, ello en torno a asegurar la sistematización de la medición realizada (Silva, 2017). La búsqueda de sistematización, sustentada en la igualdad de condiciones de administración y calificación en los instrumentos utilizados, asume la necesidad de una: "estimación justa y sistemática del aprovechamiento o habilidad del examinado en el campo de contenido que se pretende medir con la prueba y para proporcionar evidencia documentada en apoyo de las inferencias por hacer a partir de las calificaciones de la prueba" (Downing, 2012: 25).

Es decir, la sistematización buscada por los procesos psicométricos de instrumentos tales como EXANI-II, EXCOBA o PAA, pretenden contar con la evidencia necesaria para las decisiones de ingreso a las IES, a través de una medición justa de sus habilidades. En este sentido, la justicia a la que refiere el texto clásico de Downing es la igualdad formal, la isonomia. En la mirada igualitaria, un mecanismo de este tipo da por hecho el supuesto de la igualdad para resaltar el de la libertad de los aspirantes; es decir, que son iguales en condiciones para poder hacer frente libremente a las demandas que imponen estos instrumentos.

Si bien esto es un problema en sí, hay que añadir los temas de autonomía que pudieran representar el uso de mecanismos sistematizados gestionados por empresas externas. En este tema, De Garay (2020) menciona como, desde los años noventa, las universidades públicas han resaltado el riesgo a la autonomía, representado por el hecho de que instancias ajenas a las UPE funjan como medios regulatorios de procesos clave como el ingreso o el egreso. Por supuesto, esto no es sencillo, ya que las decisiones de las propias universidades no sólo deben considerar sus propios procesos, sino también las demandas sociales asociadas a sus funciones (Acosta, 2020).

Vale resaltar algunos ejercicios que las UPE implementan con fines de eliminar desigualdades (Silva, 2019) de quienes aspiran a realizar estudios superiores. Particularmente resaltan los casos de la UAA, UABCS y UAQ que, además del examen de ingreso, solicitan que los aspirantes concluyan cursos propedéuticos para poder acceder a la licenciatura de su elección. Es un mecanismo poco frecuente, sin embargo, no sólo considera que no basta con la libertad 
de participar en el proceso de selección, sino que se asume que los aspirantes tendrán necesidad de reforzar conocimientos y habilidades para el inicio de sus estudios; de esta manera, estas tres UPE se orientan, de alguna manera, a paliar diferencias previas al ingreso de estudios profesionales (Villaroel, 2014).

Existen también las UPE que aplican exámenes psicométricos o vocacionales que, si bien no fungen como factor de decisión para el ingreso, representan insumos de planeación de la trayectoria formativa de los aspirantes aceptados, en el momento que se incorporan a la vida universitaria; a este respecto, también se requiere un análisis de las características técnicas de este tipo de instrumentos para valorar la idoneidad de su aplicabilidad - elemento que trasciende el objetivo de este manuscrito. La consideración de herramientas de este tipo también puede relacionarse con la búsqueda de un acceso efectivo (Silva, 2019). Así, el reconocimiento de la posible desigualdad de las condiciones particulares de los aspirantes como elemento de planeación del ingreso abona a que los individuos tengan las mismas condiciones para realizar la forma de vida que desean (Cortés, 2005); en este caso, la posibilidad de mejorar sus condiciones en su trayecto educativo en el nivel profesional. Por supuesto, además de poner sobre la mesa los diferentes mecanismos de ingreso, es necesario considerar que hay otros elementos que permitan un trayecto formativo adecuado para los estudiantes de grupos menos favorecidos, como la gestión de becas (Tuirán, 2019).

Vale resaltar el caso de la UAGro. Esta universidad se ubica en el sur de México, en el estado de Guerrero, que es una de las entidades con entornos socioeconómicos y escolares más desfavorables de todo el país (INEE, 2019). Al igual que el resto de las UPE, la UAGro aplica un examen de ingreso, en su caso, el EXANI-II; sin embargo, su convocatoria es explícita respecto a que, del total de espacios disponibles, 13\% estarán destinados para que sean ocupados por aspirantes de grupos vulnerables (indígenas, afromexicanos guerrerenses, personas con discapacidad, residentes de la sierra de Guerrero, hijos de inmigrantes o repatriados guerrerenses) (UAGro, 2020). Esta actividad, si bien no intenta disminuir las posibles carencias de desarrollo de las condiciones sociales en las cuales los aspirantes han crecido, reconoce y asume la condición de desventaja de determinados grupos poblacionales, dejando así a un lado la perspectiva tradicional en la que las universidades asumen como ajeno el elemento de justicia social (Villalobos et al., 2017).

Existen ejemplos de universidades que no son caracterizadas como UPE, pero que realizan esfuerzos para la inclusión de grupos tradicionalmente excluidos. Tal es el caso de las universidades interculturales (Didou, 2011), o bien, la Universidad Autónoma Chapingo, que hace uso de modelos más complejos que el de la UAGro para regular el acceso mediante un mecanismo equitativo. La Universidad Autónoma Chapingo, en su modelo, considera elementos como: entidad federativa, etnia indígena, municipios pobres, calificación por escuela agropecuaria o forestal (que son perfiles afines a las licenciaturas que alberga esta institución), por telesecundaria, media regional, ingreso regional y por calificación del examen de ingreso como elementos que conforman el complejo sistema de selección (Buendía y Rivera, 2010). Por supuesto, también está el caso de la Universidad Autónoma de la Ciudad de México (UACM), la cual surgió con la finalidad de atender a sectores tradicionalmente desfavorecidos, eliminando los exámenes de ingreso y estableciendo un mecanismo azaroso para el ingreso. De acuerdo con Silva (2019), este mecanismo es complicado de operar debido a la amplia demanda y limitada oferta de espacios, además de críticas respecto a los resultados de egreso; sin embargo, como lo comenta Silva (2019), ha tratado de potenciar el egreso de sus estudiantes a través de estrategias compensatorias en el trayecto formativo de los estudiantes, como becas y cursos, atendiendo así acciones para atender a la igualdad sustancial más allá del mecanismo de ingreso en aislado. 
Centrando el discurso de nuevo en las 35 UPE en México, la UAGro destaca en el tema de la equidad en su mecanismo de ingreso, a través de destinar un porcentaje de la cuota de ingreso a grupos socialmente vulnerables. Ello es un reflejo de la intención de dicha universidad de abandonar la posición de neutralidad que los ejercicios meritocráticos suelen adoptar, favoreciendo así sólo a la idea liberalista y no al igualitarismo (Cortés, 2005). Con esa acción resalta el matiz de igualdad propio de la idea de equidad (Gómez, 2019), siendo coincidente con la intención articuladora que también le subyace, como lo expresa Silva (2019: 46): "la equidad significa articular los recursos y procesos educativos para ofrecer una educación de calidad a los jóvenes provenientes de los estratos sociales más desfavorecidos, donde desarrollen procesos de aprendizaje significativos que les permitan mejorar sus condiciones de vida". Si bien, no basta con asegurar el ingreso a la educación superior, el ejemplo de la UAGro es una acción importante que invita a reflexión de las universidades públicas en México.

\section{Reflexiones finales}

El marco normativo de la educación superior mexicana establece la necesidad de reflexionar respecto a la manera en que las diferentes instituciones procuran solucionar el problema del ingreso a sus aulas, atendiendo a la igualdad formal y la sustancial. Esto no es cosa sencilla, ya que la mera adopción de mecanismos basados únicamente en la meritocracia asume solamente la igualdad formal, tratando a todos los aspirantes de la misma manera. Sin embargo, en una sociedad marcada por la desigualdad de forma histórica, vale la pena retomar las perspectivas conceptuales que orientan hacia la consideración de la equidad, tomando ésta como base a los principios del igualitarismo de Dworking, en el cual el valor de la igualdad está por encima del de la libertad.

Como primer acercamiento a esta situación en las diferentes universidades del país, el proceso de ingreso a la educación superior ofrecida por las 35 UPE mexicanas se centra mayoritariamente en el uso de pruebas a gran escala, las cuales basan su quehacer en evaluar áreas que se consideran como prioritarias para estimar el éxito de los estudiantes aceptados en sus estudios profesionales. La lógica que subyace a este tipo de ejercicios refiere al uso de métodos que pretenden estar alejados de sesgos y ser lo más justos posibles (López et al., 2010; Wikström y Wikström, 2020). Pero, como se ha argumentado, esta idea remite a la justicia formal, en la que se asume que todas las personas son idénticas y se desarrollan en igualdad de circunstancias, cumpliendo con los principios ya enunciados por Rawls, en los que el valor de la libertad es condición básica para la equidad. Sin embargo, deja de lado los principios del matiz situacional que aseguraría una participación $-\mathrm{y}$, en este caso, competencia - entre iguales. Esto adquiere una singular relevancia para México, debido al factor de obligatoriedad del Estado para ofrecer educación superior (DOF, 2019a), lo cual, junto con la consideración de este nivel de estudios como un derecho (UNESCO, 2005), orilla a reflexionar que el papel de la universidad pública mexicana no puede conducirse como apolítico en este tipo de procesos, apelando a la justicia que los instrumentos a gran escala puedan ofrecer per se.

La relevancia de pensar en formas de ingreso a la educación superior que tiendan hacia la equidad es fundamental, ya que no sólo basta con anteponer la idea de libertad de participar en estos procesos - que resulta una posición de aparente neutralidad-, sino que es necesario considerar la posibilidad de anteponer el valor de la igualdad de condiciones de los aspirantes a este nivel educativo. Considerar el igualitarismo como estandarte en la implementación de procesos de ingreso a la educación superior permitirá alcanzar el matiz de decisión social que caracteriza a la equidad. En ese sentido, como lo argumentan Beltrán-Llavador et al. (2014), para que la universidad - como institución social - sea un agente transformador, necesita transformarse a ella misma. 
Sin duda, el caso de la UAGro es interesante, si bien mantiene elementos meritocráticos, asume la diversidad e inequidad social existente en su contexto. Algo similar ocurre con UAA, UABCS y UAQ que implementan cursos propedéuticos que fomentan el igualitarismo en su población de aspirantes; atendiendo a la búsqueda de equilibrar diferencias académicas para abonar a la equidad en el trayecto o permanencia de los estudiantes, no sólo en el proceso de selección. Si bien estas acciones demuestran avances en términos de ingreso equitativo a la educación superior pública, no debe perderse de vista la perspectiva de Rawls (2002: 36):

La justicia como equidad es utópica siendo realista: examina los límites de lo practicable desde un punto de vista realista, esto es, hasta qué punto puede un régimen democrático de nuestro mundo (dadas sus leyes y tendencias) lograr la completa realización de los valores políticos que le son propios, o dicho de otro modo, la perfección democrática.

Esta cita de Rawls nos invita a aclarar que la equidad, como cualquier ideal político, es utópico. Esto no quiere decir que las UPE nunca llegarán a ser equitativas, sino que el ideal mismo nos anima a seguir discutiendo formas en las cuales nos acerquemos a la perfección democrática que esperamos como sociedad. De esta manera, en la discusión de mecanismos de ingreso a la universidad pública no debemos olvidar los tres elementos fundamentales de la justicia equitativa: a) las personas son libres e iguales, b) es necesaria una cooperación social, y c) requerimos de un orden acordado entre iguales (Rawls, 2002); una argumentación basada sólo en la meritocracia o en la justicia formal resulta insuficiente.

Ejercicios internacionales sugieren comenzar con acciones tales como el control de forma estadística de aquellas variables identificadas como posible factor de sesgo en las pruebas objetivas, con el fin de evitar que los procesos de selección beneficien a poblaciones particulares; o bien, no utilizar los puntajes de pruebas como mecanismo de selección, sino como información para planeación institucional (Geiser, 2017). En este sentido, es importante que las UPE, en sus condiciones y demandas contextuales, se cuestionen estos elementos, además de lo que Eicheta y Ainscow (2011) establecen como indicadores de inclusión: ¿los sistemas de evaluación de rendimientos y procesos educativos están dispuestos de modo que supervisen la presencia, la participación y el éxito de todos los alumnos? ¿Se brinda apoyo adicional y específico que necesitan los grupos de alumnos más vulnerables?

De esta manera, es importante reconocer las limitaciones de las pruebas objetivas que asumen la justicia como estandarte, sobre todo en sistemas educativos caracterizados por desigualdades - como el caso mexicano. Este reconocimiento de las limitaciones de la perspectiva libertaria puede ayudar a reflexionar en varios sentidos: a) respecto a los mecanismos de ingreso implementados (como el caso de la UAGro ya mencionado); b) el control estadístico de los instrumentos (como apunta Geiser, 2017); c) el seguimiento a la trayectoria de estudiantes una vez que ingresan a la institución. En todo caso, las ideas de Dworking, en las que el valor de la igualdad está por encima del de la libertad, resultan fundamentales.

Una universidad pública que trabaja bajo el principio de equidad no sólo se deberá centrar en la regulación de ingreso a las aulas, sino que es necesario también atender la permanencia y el egreso (Didou, 2011; Silva, 2020). Pero, particularmente en lo que refiere a los mecanismos de ingreso, las UPE deben evitar reproducir desigualdad (Guzmán y Serrano, 2001), ya que la institución universitaria no debe considerarse como una fuente de inequidad en la población (Montero, 2010); sobre todo considerando que existe una marcada inequidad en el ingreso a la educación superior, ya que sectores poblacionales de bajos recursos no logran ingresar a este nivel de estudios (Maldonado-Maldonado y Mejía, 2018). En 
el contexto político y social actual del país es necesario replantear esquemas de selección, en función de las características propias de la sociedad (Cortés,

\section{Referencias}

Acosta, A. (2020), "Autonomía y gobierno institucional", en F. Martínez (coord.), La autonomía universitaria en la coyuntura actual, Aguascalientes, Universidad Autónoma de Aguascalientes, pp. 167-186.

Acosta, A. (2014), "El futuro de la educación superior en México", Revista Iberoamericana de Educación Superior, vol. 5, núm. 13, pp. 91-100, DOI: https://doi.org/10.22201/ iisue.20072872e.2014.13.122.

Aguilar-Nery, J. (2017), "Justicia educativa: itinerario de su historia conceptual en México", Revista Electrónica de Investigación Educativa, vol. 19, núm. 2, pp. 1-11, DOI: https://doi.org/10.24320/redie.2017.19.2.1216.

Alcántara, A. (2014), "Inclusión, equidad y cohesión social en las políticas de educación superior en México", Revista Mexicana de Investigación Educativa, vol. 19, núm. 60, pp. 213-239.

Alcántara, A. y Z. Navarrete (2014), "Inclusión, equidad y cohesión social en las políticas de educación superior en México", Revista Mexicana de Investigación Educativa, vol. 19, núm. 60, pp. 213-239.

Asociación Nacional de Universidades e Institutos de Educación Superior (ANUIES) (2020), Anuario estadístico de la educación superior. 2019-2020, México, ANUIES, http: / /www.anuies.mx/informacion-y-servicios / informacion-estadistica-de-educacion-superior / anuario-estadistico-de-educacion-superior.

ANUIES (2019), Anuario estadístico de la educación superior. 2018-2019, México, ANUIES, <http://www.anuies. $\mathrm{mx} /$ informacion-y-servicios/informacion-estadisticade-educacion-superior/anuario-estadistico-deeducacion-superior $>$.

Beltrán-Llavador, J., E. Íñigo-Bajo y A. Mata-Segreda (2014), "La responsabilidad social universitaria, un reto
2005), tratando de paliar las desigualdades sociales que tradicionalmente han definido a México.

en construcción permanente", Revista Iberoamericana de Educación Superior, vol. 5, núm. 14, pp. 3-18, DOI:https:// doi.org/10.22201/iisue.20072872e.2014.14.128 .

Benemérita Universidad Autónoma de Puebla (2020), Proceso de admisión a nivel licenciatura, https://admision. buap.mx/oferta-academica [Consulta: marzo de 2020].

Brunner, J. (2012), "La universidad en tiempos de masificación", Revista Iberoamericana de Educación Superior, vol. 3, núm. 7, pp. 130-143, DOI: https://doi. org/10.22201/iisue.20072872e.2012.7.69.

Buendía, A. (2020), "Revisitar las políticas para la educación superior en México: ¿de su agotamiento a su transformación?”, Universidades, vol. 86, pp. 35-52.

Buendía, A. y R. Rivera (2010), "Modelo de selección para el ingreso a la educación superior: el caso de la UACH", Revista de la Educación Superior, vol. 39, núm. 4, pp. 55-72.

Cendrós, J., J. Aular y N. Marcano (2008), "Equidad para ingresar al sistema de educación superior: programa API-Indígena de la Universidad de Zulia”, Laurus, Revista de Educación, vol. 14, núm. 28, pp. 273-204.

Centro Nacional para la Evaluación de la Educación Superior (Ceneval) (2019), Resultados del Examen Nacional de Ingreso a la Educación Superior en el año 2018, México, Ceneval.

Chaín, R., N. Gruz Ramírez, M. Martínez Morales y N. Jácome (2003), "Examen de selección y probabilidades de éxito escolar en estudios superiores. Estudio en una universidad pública estatal mexicana", Revista Electrónica de Investigación Educativa, vol. 5, núm. 1.

College Board (2020a), Guía de estudio. Prueba de aptitud académica, Puerto Rico, College Board, https://latam. collegeboard.org/wp-content/uploads/2020/04/ Guia_de_estudios_PAA_WEB_.pdf. 
College Board (2020b), ¿Qué es la PAA?, https://latam. collegeboard.org/paa/que-es-la-paa/ [Consulta: marzo de 2020].

Comisión Nacional para la Mejora Continua de la Educación (MEJOREDU) (2020), Indicadores nacionales de la mejora continua de la educación en México 2020, México, MEJOREDU.

Conferencia Regional de Educación Superior de América Latina y el Caribe (CRES) (2018), Conferencia Regional de Educación Superior de América Latina y el Caribe. Córdoba 2018. Resúmenes ejecutivos, Córdoba, Argentina, Universidad Nacional de Córdoba.

Cortés, A. y J. Palomar (2008), "El proceso de admisión como predictor del rendimiento académico en la educación superior", Universitas Psychologica, vol. 7, núm. 1, pp. 199-215.

Cortés, F. (2005), "Justicia y exclusión. Elementos para la formación de una concepción igualitaria de la justicia", en J. Botero (ed.), Con Rawls y contra Rawls: una aproximación a la filosofia política contemporánea, Bogotá, Universidad Nacional de Colombia, pp. 157-178.

Delgado, P., F. García y L. Lara (2018), "El índice académico y su impacto en los resultados de los exámenes de ingreso a la educación superior", Mendive. Revista de educación, vol. 16, núm. 1, pp. 110-121.

Diario Oficial de la Federación (DOF) (2019a), Decreto por el que se reforman, adicionan y derogan diversas disposiciones de los artículos 3o., 31 y 73 de la Constitución Política de los Estados Unidos Mexicanos, en materia educativa, https:// dof.gob.mx/nota_detalle.php?.codigo $=5560457 \& \mathrm{fec}$ ha $=15 / 05 / 2019$ [Consulta: enero de 2020].

DOF (2019b), Decreto por el que se expide la Ley General de Educación y se abroga la Ley General de la Infraestructura Física Educativa, http://www.diputados.gob.mx/LeyesBiblio/ ref/lge/LGE_orig_30sep19.pdf

De Garay, A. (2020), "La autonomía universitaria y los estudiantes", en F. Martínez (coord.), La autonomía universitaria en la coyuntura actual, Aguascalientes, Universidad Autónoma de Aguascalientes, pp. 85-102.

Didou, S. (2011), "Cobertura y promoción de la equidad en el sistema de educación superior en México.
¿Cambio de política o de retórica?”, Perfiles educativos, vol. 33, núm. Especial, pp. 59-65.

Donoso, S. y G. Hawes (2000), "El sistema de selección de alumnos de las universidades chilenas: discusión de sus fundamentos, resultados y perspectivas", Educaction Policy Analysis Archives, vol. 8, núm. 21, pp. 109-126.

Downing, S. (2012), "Doce pasos para el desarrollo de pruebas eficaces", en S. Downing y T. Haladyna (coords.), Manual para el desarrollo de pruebas a gran escala, México, Ceneval, pp. 25-57.

Echeita, G. y M. Ainscow (2011), "La educación inclusiva como derecho. Marco de referencia y pautas de acción para el desarrollo de una revolución pendiente", Tejuelo: Revista de Didáctica de la Lengua y la Literatura, vol. 12, núm. 1, pp. 26-46.

Echeita, G. y C. Duk (2008), "Inclusión educativa", Revista Electrónica Iberoamericana sobre Calidad, Eficacia y Cambio en Educación, vol. 6, núm. 2, pp. 1-8.

Enciso-Ávila, M. y J. Planas-Coll (2018), “¿Aumentar la cobertura aumenta la equidad? El caso de la educación superior en México de 1990 a 2010", Revista Iberoamericana de Educación Superior, vol. 9, núm. 25, pp. 3-23, DOI: https://doi.org/10.22201/ iisue.20072872e.2018.25.275 .

Felicetti, V. L. y A. F. Cabrera (2018), "Acesso à educação superior: O ProUni em foco", Archivos Analíticos de Políticas Educativas, vol. 26, núm. 39, DOI: http:// dx.doi.org/10.14507/epaa.26.3289.

Gallego, J. y T. Bullemore (2016), Igualitarismo. Una discusión necesaria, Santiago, Chile, Centro de Estudios Públicos.

García, I. (2016), "Patrones de elección de los participantes del concurso para el ingreso a la educación media superior de la COMIPEMS", Revista Mexicana de Investigación Educativa, vol. 21, núm. 68, pp. 95-118.

Geiser, S. (2017), "Norm-referenced tests and race-blind admissions: the case of eliminating the SAT and ACT at the University of California", California, Center for Studies in Higher Education, https://eric.ed.gov/?id=ED580807.

Gómez, J. (2019), "Una interpretación de la equidad bajo los conceptos griegos de la isonomia y la epikeia", Revista del Instituto de la fudicatura Federal, núm. 48, pp. 85-103. 
Guzmán, C. y O. Serrano (2011), "Las puertas del ingreso a la educación superior: el caso del concurso de selección a la licenciatura de la UNAM", Revista de la Educación Superior, vol. 40, núm. 157, pp. 31-53.

Hernández, M., E. Ramírez y S. Gamboa (2018), "La implementación de una evaluación estandarizada en una institución de educación superior", Innovación educativa, vol. 18, núm. 76, pp. 149-170.

Hlalele, D. y G. Alexander (2012), "University access, inclusion and social justice", South African Fournal of Higher Education, vol. 26, núm. 3, pp. 487-502.

Instituto Nacional para la Evaluación de la Educación (INEE) (2019), Panorama Educativo de México 2018. Indicadores del Sistema Educativo Nacional. Educación básica y media superior, México, INEE.

INEE (2014), El derecho a una educación de calidad. Informe 2014, México, INEE.

Instituto Tecnológico de Sonora (2020), Proceso de admisión, https://www.itson.mx/aspirantes/admisiones/ Paginas/proceso.aspx [Consulta: marzo de 2020].

Jia, Q. y D. Ericson (2016), "Equity and access to higher education in China: lessons from Hunan province for university admissions policy", International Fournal of Educational Development, núm. 52, pp. 97-110.

Jiménez, A. (2017), "Una mirada hacia la calidad de la educación primaria en Baja California, México: marginación escolar y equidad en sus resultados", Revista Iberoamericana sobre Calidad, Eficacia y Cambio en la Educación, vol. 15, núm. 3, pp. 35-48, DOI: https:// doi.org/10.15366/reice2017.15.3.002 .

Lima, M., A. Ávila y C. López (2015), “Influencia de los antecedentes académicos para el ingreso en una universidad pública mexicana: un estudio de caso", Revista Iberoamericana sobre Calidad, Eficacia y Cambio en Educación, vol. 13, núm. 3, pp. 107-119.

López, I., G. Echazarreta, S. Pech y B. Gómez (2010), "Selección y permanencia en la educación superior: el caso de la Universidad Autónoma de Yucatán”, Revista Iberoamericana de Evaluación Educativa, vol. 3, núm. 2, pp. 90-102.

Maldonado-Maldonado, A. y G. Mejía (2018), "Higher education systems and institutions, Mexico", en C. Shin y P. Teixeira (eds.), Encyclopedia of international Higher Education Systems and Institutions, Netherlands, Springer, pp. 1-12.

Maldonado-Maldonado, A. y J. González (2017), "Educación superior en América Latina: dime qué tan estratificado está tu sistema y te diré qué tan público eres", Ponencia presentada en el XIV Congreso Nacional de Investigación Educativa, San Luis Potosí, México.

Martínez, F. (2020), "Autonomía universitaria: antecedentes, retos y perspectivas", en F. Martínez (coord.), La autonomía universitaria en la coyuntura actual, Aguascalientes, Universidad Autónoma de Aguascalientes, pp. 25-71.

Mendoza, R. (2018), "Inclusión como política educativa: hacia un sistema educativo único en un México cultural y lingüísticamente diverso", Sinéctica, vol. 50, pp. 1-16.

Mendoza-Rojas, J. (2015), "Ampliación de la oferta de educación superior en México y creación de instituciones públicas en el periodo 2001-2012", Revista Iberoamericana de Educación Superior, vol. 6, núm. 16, pp. 3-32, DOI: https:// doi.org/10.22201/iisue.20072872e.2015.16.149.

Métrica educativa (2020), Examen de Habilidades y Conocimientos Básicos (EXHCOBA), https://metrica.edu. mx/examenes/exhcoba/ [Consulta: marzo de 2020].

Michel, R. y S. Pollard (2020), "An overview of higher education admission processes", en M. Oliveri y C. Wendler (eds.), Higher education admission practices, Cambridge, Cambridge University Press, pp. 5-17.

Montero, E. (2010), "Excelencia y equidad en pruebas de admisión: una propuesta emergente para la universidad de Costa Rica", Actualidades Investigativas en Educación, vol. 10, núm. 2, pp. 1-19, DOI: https://doi.org/10.15517/ aie.v10i2.10119.

Niessen, A. y R. Meijer (2020), "Character-based admissions criteria in the United States and Europe: rationale, evidence, and some critical remarks", en M. Oliveri y C. Wendler (eds.), Higher education admission practices, Cambridge, Cambridge University Press, pp. 76-98.

Ordorika, I. (2014), "Renovar las políticas de acceso a la educación superior" [Editorial], Revista de la Educación Superior, vol. 43, núm. 170, DOI: https://doi.org/10.1016/j. resu.2015.02.001 
Piñero, S., S. Juárez y R. Chaín (2011), "Modelo de regresión para el desempeño en el EXAN-II de los aspirantes a la Universidad Veracruzana", Ponencia presentada en el XI Congreso Nacional de Investigación Educativa, México.

Rawls, J. (2002), La justicia como equidad. Una reformulación, Barcelona, Paidós.

Rodríguez, C. y G. Padilla (2017), "Elección profesional y sesgo de selección: evaluación de los sistemas de admisión universitaria en Chile en un contexto de agenda pro-inclusión”, Avaliação: Revista da Avaliação da Educação Superior (Campinas), vol. 22, núm. 3, pp. 852-870, DOI: https://doi.org/10.1590/s141440772017000300015 .

Rodríguez-Gómez, R. (2015), "Educación superior y desarrollo. La importancia del contexto local", en R. Cordera, M. Flores y M. Fuentes (coords.), México social: regresar a lo fundamental, México, Universidad Nacional Autónoma de México, pp. 199-213.

Salas, I., A. Buendía y A. Pérez (2019), "La apropiación de la política educativa en las universidades públicas mexicanas: entre la ambigüedad y la diversidad", Revista de la Educación Superior, vol. 48, núm. 191, pp. 25-49.

Saldaña, J. (2013), The coding manual for qualitative researchers, California, Sage.

Secretaría de Educación Pública (SEP) (2019), Programa Sectorial de Educación 2019-2023, México, SEP.

SEP (2014), Diagnóstico del programa. Inclusión y equidad educativa, México, SEP, https://www.inclusionyequidad. sep.gob.mx/files/transparencia/diagnostico/ Diagnostico_S244.pdf .

Segrera, F. L. (2015), "Educación superior comparada: tendencias mundiales y de América Latina y Caribe," Avaliação: Revista da Avaliação da Educação Superior, vol. 21, núm. 1, pp. 13-32, DOI: https://doi.org/10.1590/ s1414-40772016000100002

Silva, C. (2017), "De discursos e incongruencias de la evaluación en México", en P. Gutiérrez, B. Anguiano y C. Villapando (coords.), Investigar y evaluar en educación, Andalucía, Círculo Rojo, pp. 101-142.
Silva, M. (2020), "La dimensión pedagógica de la equidad en educación superior", Archivos Analíticos de Políticas Educativas, vol. 28, núm. 46, DOI: https://doi. org/10.14507/epaa.28.5039

Silva, M. (2019), "Tres iniciativas de equidad educativa universitaria en la Ciudad de México", Revista Mexicana de Investigación Educativa, vol. 24, núm. 80, pp. 43-68.

Silva-Laya, M. (2012), "Equidad en la educación superior en México: la necesidad de un nuevo concepto y nuevas políticas", Archivos Analíticos de Politicas Educativas, vol. 20, núm. 4, DOI: https://doi.org/10.14507/epaa. v20n4.2012.

Suárez, G. y J. Gairín (2018), "Equidad y educación superior", en J. Gairín y G. Palmeros (coords.), Políticas y prácticas para la equidad en educación superior, Madrid, Wolters Kluwer, pp. 27-37.

Tapia, L. (2016), "El subsistema de universidades interculturales en México. Entre la política social y la política educativa", Revista Latinoamericana de Estudios Educativos, vol. 46, núm. 1, pp. 7-50.

Tuirán, R. (2019), "La educación superior: promesas de campaña y ejercicio de gobierno", Revista de la Educación Superior, vol. 48, núm. 190, pp. 113-183.

Torrance, H. (2017), "Blaming the victim: assessment, examinations, and the responsibilisation of students and teachers in neo-liberal governance", Discourse: Studies in the Cultural Politics of Education, vol. 38, núm. 1, pp. 83-96, DOI: 10.1080/01596306.2015.1104854.

UNESCO (2005), Hacia las sociedades del conocimiento, París, UNESCO.

Universidad Autónoma "Benito Juárez" de Oaxaca (2020), Proceso de admisión a nivel superior, http://www. admision.uabjo.mx/nivel/superior [Consulta: marzo de 2020].

Universidad Autónoma de Aguascalientes (2020), Convocatoria de admisión. Licenciaturas e ingenierías 20202021, https://www.uaa.mx/portal/wp-content/ uploads/2020/02/INFORMACI\%C3\%93NG E N E R A L - D E - A D M I S I \% C 3\% 93 N LICENCIATURA-2020-1-1.pdf [Consulta: marzo de 2020]. 
Universidad Autónoma de Baja California (2020), Convocatoria concurso de selección para el ingreso a licenciatura 2020-2 2021-1, https://drive.google.com/file/d/1ErYnr3lw6PDtQRToJlkmWtPjojXebOZ/view [Consulta: marzo de 2020].

Universidad Autónoma de Baja California Sur (2020), Convocatoria al concurso de selección de alumnos de nuevo ingreso al periodo 2020-II, http://uabcs.mx/secciones/ contenido/3180 [Consulta: marzo de 2020].

Universidad Autónoma de Campeche (2020), Concurso de ingreso. Convocatoria ciclo escolar 2020-2021, https:// drive.google.com/file/d/1 r-D4go9YxxAkhEqThU4 FAFRVIrvduAFe/view [Consulta: marzo de 2020].

Universidad Autónoma de Chiapas (2020), Convocatoria de admisión para carreras presenciales y a distancia. Enero-junio 2020, http://cedes.unach.mx/index.php/component/ k2/item/146-convocatoria-enero-junio-2020 [Consulta: marzo de 2020].

Universidad Autónoma de Chihuahua (2020), Proceso de admisión, https://uach.mx/aspirantes/proceso-deadmision/ [Consulta: marzo de 2020].

Universidad Autónoma de Ciudad Juárez (2020), Proceso de admisión para nivel licenciatura, http://www.uacj.mx/ admision/licenciaturas/index.html [Consulta: marzo de 2020].

Universidad Autónoma de Coahuila (2020), Convocatoria para ingreso a nivel licenciatura, http://148.212.9.10/ aspirantes2/documen/convocatoria.pdf [Consulta: marzo de 2020].

Universidad Autónoma de Guerrero (2020), Convocatoria para participar en el examen de admisión para el ciclo escolar 2019-2020, http://dae.uagro.mx/regweb/descargas/ ConvocatoriaNSuperior2019-2020.pdf [Consulta: marzo de 2020].

Universidad Autónoma de Nayarit (2020), Convocatoria para el proceso de ingreso 2020, https://admision.uan.mx/ [Consulta: marzo de 2020].

Universidad Autónoma de Nuevo León (2020), Concurso de ingreso a licenciatura, https://www.uanl.mx/tramites/ concurso-de-ingreso-a-licenciatura/ [Consulta: marzo de 2020].
Universidad Autónoma de Occidente (2020), Convocatoria al proceso de admisión a licenciatura. Ciclo escolar 2020-2021, https://uadeo.mx/aspirante/images/Convocatorias/ convocatoria-admision-2020-2021.jpg [Consulta: marzo de 2020].

Universidad Autónoma de Querétaro (2020), Convocatoria para el proceso de selección y admisión a nivel licenciatura enero-junio 2020, https://dsa.uaq.mx/convocatorias/ ConvocatoriaLic2019-PUBLICADA-I.pdf [Consulta: marzo de 2020].

Universidad Autónoma de San Luis Potosí (2020), Instructivo para examen de admisión 2020-2021, http:// www.uaslp.mx/admisiones/Paginas/instructivo.aspx [Consulta: marzo de 2020].

Universidad Autónoma de Sinaloa (2020), Convocatoria para ingresar a estudios de nivel profesional, ciclo escolar 20202021, https://www.uas.edu.mx/preinscripcion2020/ CONVOCATORIA_ADMISION_2020.pdf [Consulta: marzo de 2020].

Universidad Autónoma de Tamaulipas (2020), Proceso de inscripción, https://www.uat.edu.mx/Paginas/ Inscripci $\% \mathrm{C} 3 \% \mathrm{~B} 3 \mathrm{n} /$ procesodeinscripcion.aspx [Consulta: marzo de 2020].

Universidad Autónoma de Tlaxcala (2020), Ingreso a licenciaturas 2020-2021, https://uatx.mx/admision/ index.php?pdf=/admision $/$ admision 2020-4-3.pdf [Consulta: marzo de 2020].

Universidad Autónoma de Yucatán (2020), Aspirantes a nivel licenciatura, https://www.uady.mx/aspirantes.php [Consulta: marzo de 2020].

Universidad Autónoma de Zacatecas (2020), Proceso único de ingreso, http://www.escolar.uaz.edu.mx/ documents / 10181/13461/Convocatoria+general /0653b8eb-f2c7-4952-9ed3-eb0bc77240ac [Consulta: marzo de 2020].

Universidad Autónoma del Carmen (2020), Convocatoria para cursar estudios de tipo superior en modalidad escolarizada, http://www.unacar.mx/preinscripciones_2020/ licenciatura/index.html [Consulta: marzo de 2020].

Universidad Autónoma del Estado de Hidalgo (2020), Convocatoria Licenciatura Fulio-Diciembre 2020, https:// 
www.uaeh.edu.mx/administracion_escolar / documentos/julio-diciembre-2020/convocatorialicenciatura-2020.pdf [Consulta: marzo de 2020].

Universidad Autónoma del Estado de México (2020), Convocatoria para nuevo ingreso 2020, http:/ / nuevoingreso. uaemex.mx/convocatorias/ep.pdf [Consulta: marzo de 2020].

Universidad Autónoma del Estado de Morelos (2020), Convocatoria para ingresar a estudios de nivel superior, https:// www.uaem.mx/convocatoria-nivel-superior-2020/ [Consulta: marzo de 2020].

Universidad de Colima (2020), Convocatoria para el proceso de admisión para ingresar a las carreras de nivel licenciatura en el ciclo escolar 2020-2021, https://cenedic.ucol.mx/content/ modulos/convocatorias/227/file/Convocatoria_ Licenciatura_2020.pdf [Consulta: marzo de 2020].

Universidad de Guadalajara (2020), Procedimiento para el concurso de ingreso, http://www.udg.mx/es/aspirantes [Consulta: marzo de 2020].

Universidad de Guanajuato (2020), Convocatoria proceso de admisión agosto-diciembre 2020, https://www.ugto. $\mathrm{mx} /$ admision/images/admision-licenciaturas-agosto2020-universidad-guanajuato-ug-ugto.pdf [Consulta: marzo de 2020].

Universidad de Quintana Roo (2020), Convocatoria admisiones otoño 2020 y primavera 2021, http:/ / www.uqroo. $\mathrm{mx} /$ admisiones/ConvocatoriaAdmisionesOto2020Prim2021\%282\%29.pdf [Consulta: marzo de 2020].

Universidad de Sonora (2020), Convocatoria para programas presenciales, https://admision.unison.mx/convocatoria_ presencial2020.pdf [Consulta: marzo de 2020].

Universidad Juárez Autónoma de Tabasco (2020), Proceso de ingreso, http://www.ujat.mx/aspirantes [Consulta: marzo de 2020].
Universidad Juárez del Estado de Durango (2020), Aspirantes, https://www.ujed.mx/aspirantes [Consulta: marzo de 2020].

Universidad Michoacana de San Nicolás de Hidalgo (2020), Convocatoria para el ingreso a nivel superior, https:/ / www.siia.umich.mx/escolar/convocatoria2021/ ConvocatoriaLic.html [Consulta: marzo de 2020].

Universidad Veracruzana (2020), Proceso de ingreso a la Universidad Veracruzana, https://www.uv.mx/escolar/ licenciatura2020/convocatorias/index.html [Consulta: marzo de 2020].

Vera, H. y M. González-Ledesma (2018), "Calidad y evaluación: matrimonio del cielo y el infierno", Perfiles Educativos, vol. 40, núm. Especial, pp. 53-97, DOI: https://doi.org/10.22201/iisue.24486167e.2018. especial.59180 .

Villalobos, C., E. Treviño, I. Wyman y J. Scheele (2017), "Social justice debate and college access in Latin America: merit or need? The role of educational institutions and states in broadening access to higher education in the region", Archivos Analíticos de Politicas Educativas, vol. 25, núm. 73, DOI: http://dx.doi. org/10.14507/epaa.25.2879

Villaroel, J. (2014), "Epistemología de las pruebas psicológicas para el ingreso a la universidad", Sophia: colección de filosofía de la educación, vol. 16, núm. 1, pp. 249-274, DOI: https://doi.org/10.17163/soph. $\mathrm{n} 16.2014 .11$

Wikström, G. y M. Wikström (2020), "Merit-based admissions in higher education en M. Oliveri y C. Wendler (eds.), Higher education admission practices, Cambridge, Cambridge University Press, pp. 34-50.

\section{Cómo citar este artículo:}

Jiménez-Moreno, José-Alfonso (2021), "Mecanismos de ingreso de las universidades públicas estatales en México: un análisis bajo el concepto de equidad", Revista Iberoamericana de Educación Superior (RIES), vol. XII, núm. 35, pp. 3-27, DoI: https://doi.org/10.22201/ iisue.20072872e.2021.35.1080 [Consulta: fecha de última consulta]. 